\title{
Ossifying fibroma of maxilla masquerading as Chondroblastoma
}

\author{
Raman Wadhera ${ }^{1}$, Sahana. $\mathbf{P}^{2, *}$, Aarushi Vashist ${ }^{3}$, Usha Sehrawat ${ }^{4}$ \\ ${ }^{1}$ Professor, ${ }^{2,3}$ Junior Resident, ${ }^{4}$ Private Practitioner, Dept. of ENT, Pandit Bhagwat Dayal Sharma Post Graduate Institute of Medical \\ Sciences, Rohtak, Haryana, India
}

*Corresponding Author: Sahana. P

Email: sahanaponnuvelu@gmail.com

\begin{abstract}
Ossifying fibroma form a part of the group of lesions called fibro-osseous lesions of the jaws. They are rare, benign, non-aggressive tumours commonly seen in head and neck region. They arise from a layer of fibrous connective-tissue surrounding the roots of teeth. This layer contains multipotential cells that are capable of forming cementum, lamellar bone, and fibrous tissue. The lesion is generally asymptomatic until the growth produces pain, paresthesias, and facial asymmetry. After radiological imaging complete surgical excision remains the treatment of choice followed by histopathological confirmation. This paper presents the case of a 13 year old male who presented with asymptomatic swelling of face, which was radiologically diagnosed as chondroblastoma of maxilla, turned out to be ossifying fibroma after histopathological examination. This paper emphasizes the need for correlation of clinical, radiological and histological findings before confirming the diagnosis.
\end{abstract}

Keywords: Ossifying fibroma, Fibro-osseous lesion, Chondroblastoma, Maxilla.

\section{Introduction}

Fibro-osseous lesions represent a group of diseases in which the normal bone tissue is replaced by fibroblasts and fibrous tissue, with varying amounts of mineralized material in it. ${ }^{1}$ Fibro-osseous lesions include fibrous dysplasia, osseous dysplasia, ossifying fibroma, central giant cell granuloma, cherubism and bone cyst. While some of these lesions are neoplastic, others are due to metabolic disturbances and cause considerable diagnostic challenge.

Ossifying fibroma is a rare form of fibro-osseous lesion that arises from craniofacial skeleton. It can affect both the mandible and the maxilla, most commonly the mandible. Juvenile ossifying fibroma also called as Aggressive or Active ossifying fibroma is a more aggressive form of ossifying fibroma arising in children less than 15 years of age. $^{2}$

The usual presentation of the tumor is a slow growing mass and is usually asymptomatic. ${ }^{3}$ A definitive diagnosis is made after correlation of clinical, radiological and histological finding. Here we report a case of 13 year old male with ossifying fibroma of left maxilla.

\section{Case Report}

A 13 year old male presented to ENT OPD with chief complaints of gradually increasing swelling in left cheek of 1 year duration (Fig. 1a and 1b). There was no history of pain or difficulty in vision or any nasal symptoms like nasal blockade or discharge. History of trauma to face was ruled out.

On examination, there was facial asymmetry on the left side cheek due to a diffuse swelling measuring approximately $6 \times 4 \mathrm{~cm}$ with ill-defined borders. On palpation, firm to hard, non-tender mass was palpable below left eye in maxillary region. There was no signs of inflammation or discharge over the swelling and the skin over the swelling was mobile. Vision was unaffected and eye movements were normal in all directions.
There was no significant lymphadenopathy in cervical and supraclavicular region. All the baseline investigations like complete hemogram, liver function test, renal function test, X-ray chest and USG abdomen were well within normal limits.

CT Paranasal sinuses showed large well defined minimal to non-enhancing expansile lytic lesion with sclerotic margin and narrow zone of transition involving the maxilla on left side and floor of left maxillary sinus. The lesion caused thinning of floor of left orbit with small component of lesion projecting in the extraconal space of the left orbit, however no obvious cortical breach is seen. Multiple scattered calcification- Ring and Arc type (Fig. 2a) and popcorn type (Fig. 2b) is seen within the lesion. A radiological provisional diagnosis of Chondroblastoma was made (Coronal sectionFig. 2a and 2b, Axial section- Fig. 2c and 2d)

Later surgical excision of the mass was done under general anaesthesia using a Weber-Ferguson approach (Fig. $3 a)$. This procedure allowed en bloc resection of the mass with preservation of the orbital periosteum. The specimen $(9 \times 5 \times 4 \mathrm{~cm})$ removed was sent for histopathological examination (Fig. 3b). The patient was observed in hospital for 1 week and was discharged later under cover of antibiotics and analgesics and was called for follow up after 1 week.

Biopsy report showed well circumscribed fibrous connective tissue with mineralization areas and thus confirming ossifying fibroma.

The follow up was uneventful and 3 month follow up showed no signs of recurrence. 


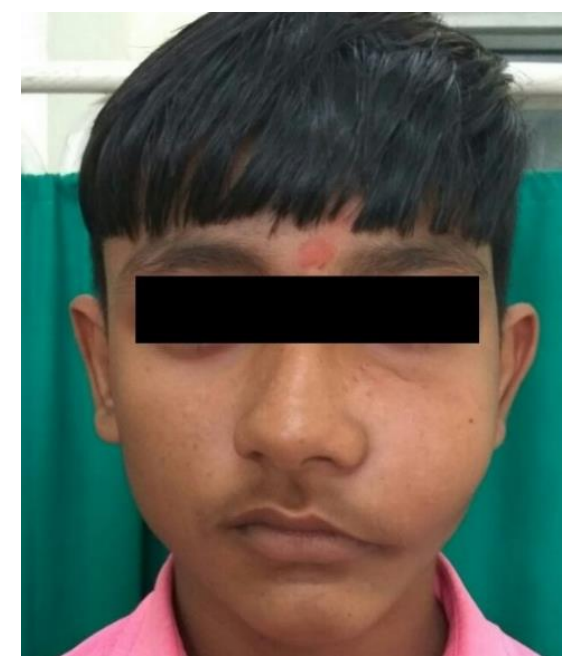

Fig. 1a: Swelling in left cheek

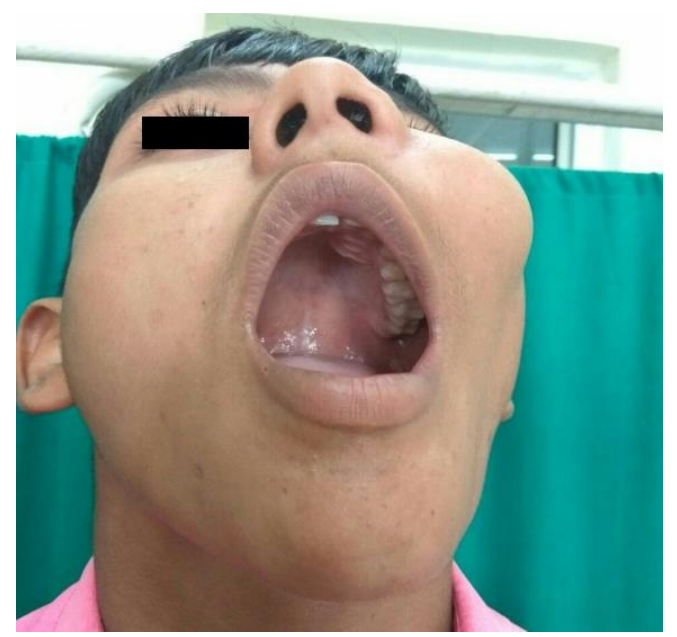

Fig. 1b: Swelling in left cheek

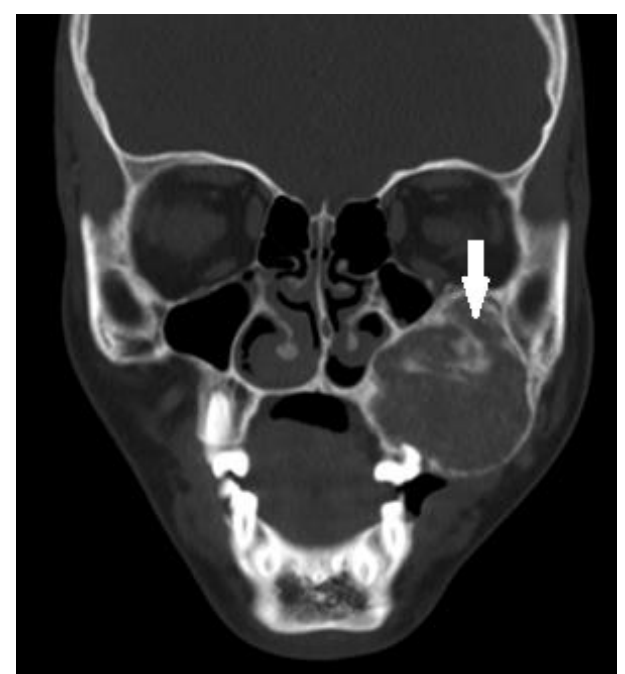

Fig. 2a: CT PNS- Coronal section showing Ring and arc calcification indicated by arrow.

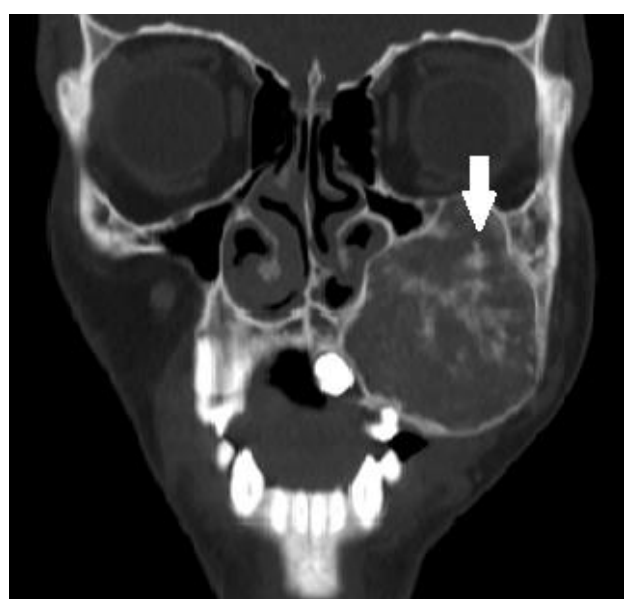

Fig. 2b: CT PNS- Coronal section showing Popcorn calcification indicated by arrow.

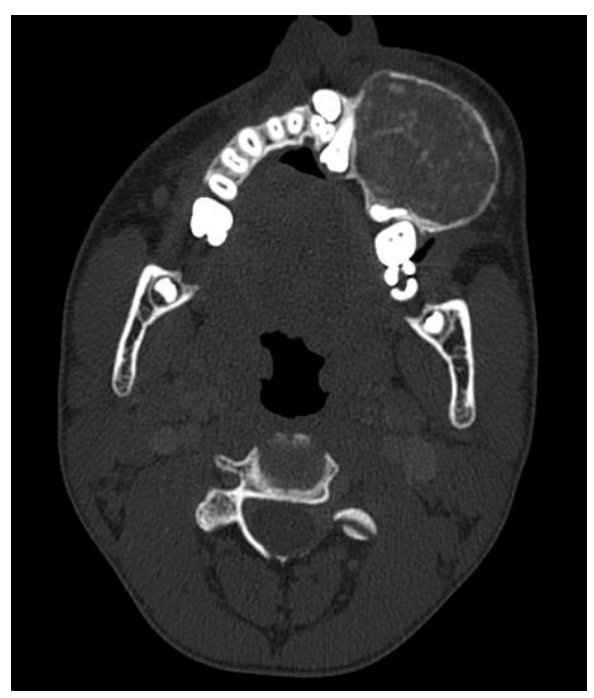

Fig. 2c: CT PNS- Axial section showing well defined minimal to non-enhancing expansile lytic lesion with sclerotic margin in left maxilla

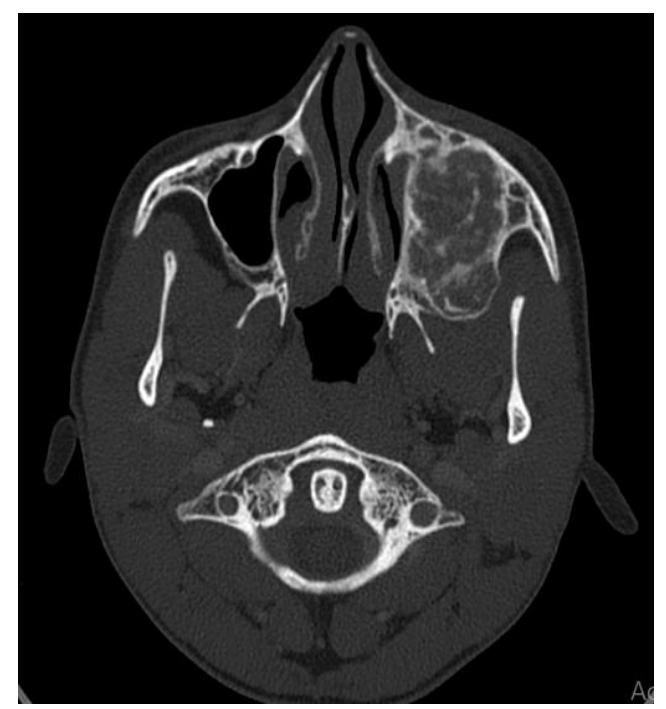

Fig. 2d: CT PNS- Axial section showing calcification in the lesion 


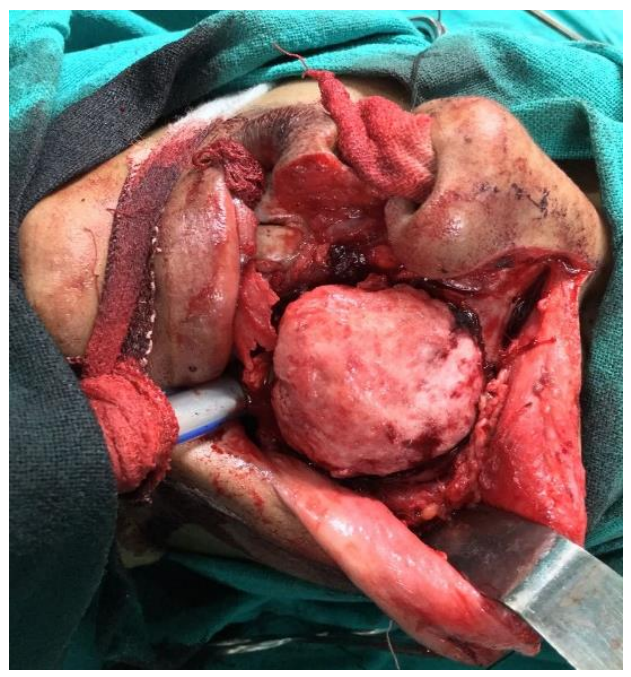

Fig. 3a: Surgical excision of mass using Weber-Ferguson approach

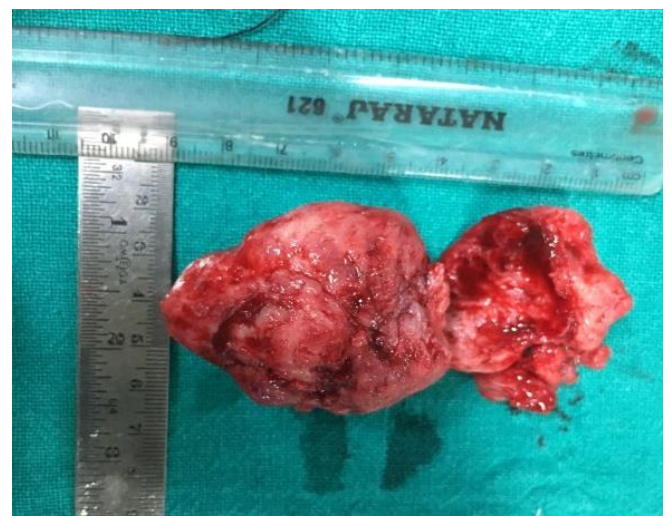

Fig. 3b: Excised mass of $9 \times 5 \times 4 \mathrm{~cm}$

\section{Discussion}

Ossifying fibromas are well circumscribed slow growing lesions which are generally asymptomatic. The lesions are most commonly seen in the second to fourth decades of life with female preponderance. ${ }^{4}$ Ossifying fibroma are most commonly monostotic. In less than 5\% cases more than one bone involvement occurs, with its most common occurrence in cranial bones. Among the cranial bones mandible is commonly involved in the premolar-molar region and approximately $30 \%$ cases are found in the maxilla.

The most common presentation is painless bone swelling, ${ }^{5}$ and this is supported by our present case. The lesion in maxilla may lead to cortical expansion with obliteration of gingivobuccal sulcus and its extension into orbital floor and nasal cavity may lead to epiphora and epistaxis respectively. ${ }^{6}$

Although the cell of origin for ossifying fibroma is unknown, it may derive from elements present in the periodontal ligament space. ${ }^{7}$

Radiologically both ossifying fibroma and chondroblastoma are well circumscribed lesion with sclerotic margins. Calcification can be present in both the lesions. There is a very little difference in the pattern of calcification and hence may lead to misdiagnosis.
The characteristic appearance of mineralized chondroid matrix is a ring-and-arc pattern of calcification. This characteristic chondroid calcification usually allows confident radiologic diagnosis of a cartilaginous lesion and is often the most dominant feature. ${ }^{8}$ In our case this lead to the radiologic confusion leading to the diagnosis of chondroblastoma.

In contrast ossifying fibroma has a more dense homogenous amorphous calcification which was radiologically absent in our case. ${ }^{9}$

Histologically ossifying fibromas are cellular, relatively avascular, having dense fibrous stroma. There are scattered calcified deposits of trabeculae of woven or lamellar bone and also have deposits of cementum like substances and basophilic staining. ${ }^{7}$ Whereas in chondroblastoma, there is an admixture of mononuclear chondroblasts and multinucleated osteoclast-type giant cells thus leading to histopathological differentiation between ossifying fibroma and chondroblastoma.

Ossifying fibroma should be differentiated from other fibro-osseous lesions which pose a diagnostic challenge.

Radiologically fibrous dysplasia has uniform pattern of calcification which may show the classic ground glass appearance, absent in ossifying fibroma. Histological differentiation between ossifying fibroma and fibrous dysplasia can be made based on the variation in the arrangement of fibrous stroma which is haphazard in the former, compared to whorled pattern in the later. ${ }^{10}$

Juvenile ossifying fibroma shows more aggressive clinical behaviour and more cellular stroma.

Ossifying fibroma can be differentiated from the sarcomas and carcinomas by their well-defined border.

As there is high chance of recurrence, treatment of choice for ossifying fibroma is complete surgical resection. ${ }^{5,10}$

\section{Conclusion}

There are many features in common between ossifying fibromas and other benign lesions, posing diagnostic challenge. In our case though radiological diagnosis of chondroblastoma was made, histological confirmation proved it to be ossifying fibroma. Hence correlation of clinical, radiological and histological findings has to be done before confirming the diagnosis.

Ossifying fibromas, though are benign, asymptomatic lesions they should be treated earlier because of its aggressive nature. As the lesions have a high recurrence rate, long term follow-up is required in these patients.

\section{Source of Support: None}

\section{Conflict of Interest: None}

\section{References}

1. Gondivkar SM, Gadbail AR, Chole R, Parikh RV, Balsaraf S. Ossifying fibroma of the jaws: report of two cases and literature review. Oral Oncol 2011;47:804-809.

2. Sun G, Chen X, Tang E, Li Z, Li J. Juvenile ossifying fibroma of the maxilla. Int J Oral Maxillofac Surg 2007;36(1):82-85. 
3. Kashyap RR, Naik GR, Gogineni SB. Asymptomatic presentation of aggressive ossifying fibroma: A case report. Case Rep Dent 2011;2011:523751.

4. Alsharif MJ, Sun ZJ, Chen XM, Wang SP, Zhao YF. Benign fibro-osseous lesions of the jaws: a study of 127 Chinese patients and review of the literature. Int J Surg Pathol 2009; 17:122-134.

5. Brannon RB, Fowler CB. Benign fibro-osseous lesions: a review of current concepts. Adv Anat Pathol 2001;8:126-143.

6. Agarwal SP, Kumar S, Singh HP, Usmani SA. Huge ossifying fibroma maxilla. Natl J Maxillofac Surg 2015;6:241-243.

7. Mohammadi-Araghi H, Haery C. Fibro-osseous lesions of craniofacial bones. The role of imaging. Radiol Clin North Am 1993;31:121-134.

8. Murphey MD, Walker EA, Wilson AJ, Kransdorf MJ, Temple HT, Gannon FH. From the Archives of the AFIP: imaging of primary chondrosarcoma: radiologic-pathologic correlation Radio Graphics 2003;23(5):1245-1278.

9. Juhl J, Crummy AB (1987) Paul and Juhl's Essentials of Radiologic Imaging. In: Bone tumours and related conditions, $5^{\text {th }}$ edn. Lippincott, Philadelphia, pp 131-77.

10. Waldron CA. Fibro-osseous Lesions of the Jaws. J Oral Maxillofac Surg 1985;43:249-262.

How to cite this article: Wadhera R, Sahana. P, Vashist A, Sehrawat U. Ossifying fibroma of maxilla masquerading as Chondroblastoma. Indian J Anat Surg Head Neck Brain 2018;4(4):123-126. 